\title{
Collective lattice resonances in disordered and quasi-random all-dielectric metasurfaces
}

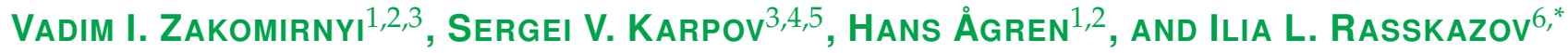 \\ ${ }^{1}$ Department of Theoretical Chemistry and Biology, School of Engineering Sciences in Chemistry, Biotechnology and Health, Royal Institute of Technology, \\ Stockholm, SE-10691, Sweden \\ ${ }^{2}$ Federal Siberian Research Clinical Centre under FMBA of Russia, Krasnoyarsk, 660037, Russia \\ ${ }^{3}$ Institute of Nanotechnology, Spectroscopy and Quantum Chemistry, Siberian Federal University, Krasnoyarsk 660041, Russia \\ ${ }^{4}$ Siberian State University of Science and Technology, Krasnoyarsk, 660014, Russia \\ ${ }^{5}$ Kirensky Institute of Physics, Federal Research Center KSC SB RAS, 660036, Krasnoyarsk, Russia \\ ${ }^{6}$ The Institute of Optics, University of Rochester, Rochester, NY 14627, USA \\ *Corresponding author: irasskaz@ur.rochester.edu
}

Compiled March 7, 2019

\begin{abstract}
Collective lattice resonances in disordered $2 D$ arrays of spherical Si nanoparticles (NPs) have been thoroughly studied within the framework of the coupled dipole approximation. Three types of defects have been analyzed: positional disorder, size disorder, and quasi-random disorder. We show that the positional disorder strongly suppresses either the electric dipole (ED) or the magnetic dipole (MD) coupling depending on the axis along which the NPs are shifted. Contrary, size disorder strongly affects only the MD response, while the the ED resonance can be almost intact, depending on the lattice configuration. Finally, random removing of NPs from an ordered $2 D$ lattice reveals a quite surprising result: hybridization of the ED and MD resonances with lattice modes remains observable even in the case of random removing of up to $84 \%$ of the NPs from the ordered array. Reported results could be important for rational design and utilization of metasurfaces, solar cells and other all-dielectric photonic devices. ๑ 2019 Optical Society of America
\end{abstract}

http://dx.doi.org/10.1364/ao.XX.XXXXXX

\section{INTRODUCTION}

Strong coupling between lattice modes in arrays of nanoparticles (NPs) and Mie-type oscillations localized within a single NP, has attracted significant attention over the last decade. Pioneering theoretical predictions for $1 D$ arrays of Ag NPs [1-3] and consequent experimental verification for $2 D$ arrays of $A u$ NPs [4-6] have given a momentum to a great number of excellent applications of collective lattice resonances in lasers [7-12], biosensors [13-18], emission enhancement [19-22], and color printing [23-26].

To the date, most of the studies have considered diffractive coupling between electric dipole (ED) oscillations and WoodRayleigh anomalies $[27,28]$ in arrays of classic plasmonic NPs like Au or Ag. However, quite recently a significant attention has been turned to alternative plasmonic materials like indium-tinoxide [29], aluminum [30-33], transition metal nitrides [22, 34, 35], and nickel [36]. The use of these materials makes it possible to tailor a wavelength of collective lattice modes within a wide spectral range, from UV [31] to IR [35], or enable a magnetooptical activity $[37,38]$ which paves the way to a rich variety of novel and promising applications.
In this context, dielectric NPs with both electric and magnetic dipole (MD) resonances [39] represent the case of specific interest. Arrays of all-dielectric NPs have already found a number of excellent applications in photonics and nanotechnology spanning light guiding [40-42], metamaterials [43], metasurfaces [44-48], mid-infrared filters [49], and others [50]. However, the coupling between localized oscillations in a single dielectric NP and lattice modes has been addressed only quite recently [51-54] with particular attention to ED and MD coupling [55] and overlapping [56] in 2D Si nanodisk arrays.

While various aspects of diffractive behaviour of ED and MD resonances in arrays of NPs have been heavily studied in the recent decade [57-60], just a few works have addressed the effects of positional and size disorders [61-63], and only for arrays with purely ED coupling. Quite interesting results have also been reported for lasing $[64,65]$ and solar energy harvesting $[62,66-68]$ in various types of quasi-periodic and aperiodic structures. However, it is a well known fact that the presence of imperfections in $1 D$ chains of NPs $[69,70], 2 D$ structures [71-76], $3 D$ metamaterials [77], and fractal aggregates [78] may lead to various intriguing effects.

In this work we thoroughly address this problem, within 
the coupled dipole approximation, and study three types of imperfections in 2D arrays of Si nanospheres: (i) disorder in positions of Si nanospheres of the same size; (ii) disorder in sizes of Si nanospheres arranged in an ordered $2 D$ lattice, and (iii) quasi-ordered $2 D$ arrays of Si nanospheres of the same size. A comprehensive analysis of these scenarios reveals different impact of disorder on ED and MD coupling with lattice modes.

The paper is organized as follows. In Sec. 2, we provide a theoretical background for the coupled dipole approximation; Next, in Sec. 3 we discuss general features of ED and MD coupling in ordered lattices; then, the impact of positional and size disorder on optical properties of $2 D$ lattices of Si NPs, as well as their quasi-random modifications are discussed in Sec. 4; Finally, we draw general conclusions in Sec. 5 .

\section{COUPLED DIPOLE APPROXIMATION}

Consider an array of $N$ spherical NPs embedded in vacuum. Under the incident planewave illumination with electric $\mathbf{E}^{0}$ and magnetic $\mathbf{H}^{0}$ components, the $i$-th particle located at $\mathbf{r}_{i}$ acquires electric $\mathbf{d}_{i}$ and magnetic $\mathbf{m}_{i}$ dipole moments which are coupled to other dipoles and to an external electromagnetic filed via the coupled dipole equations [39, 79, 80]:

$$
\left\{\begin{array}{l}
\mathbf{d}_{i}=\alpha_{i}^{e}\left(\mathbf{E}_{i}^{0}+\sum_{j \neq i}^{N} \hat{G}_{i j} \mathbf{d}_{j}-\sqrt{\frac{\mu_{0}}{\varepsilon_{0}}} \sum_{j \neq i}^{N} \hat{C}_{i j} \mathbf{m}_{j}\right), \\
\mathbf{m}_{i}=\alpha_{i}^{m}\left(\mathbf{H}_{i}^{0}+\sum_{j \neq i}^{N} \hat{G}_{i j} \mathbf{m}_{j}+\sqrt{\frac{\varepsilon_{0}}{\mu_{0}}} \sum_{j \neq i}^{N} \hat{C}_{i j} \mathbf{d}_{j}\right),
\end{array}\right.
$$

where $\alpha_{i}^{e}$ and $\alpha_{i}^{m}$ are electric and magnetic dipole polarizabilities of the $i$-th particle, respectively, $\varepsilon_{0}$ and $\mu_{0}$ are the dielectric constant and magnetic permeability of vacuum, $\mathbf{E}_{i}^{0}=\mathbf{E}^{0}\left(\mathbf{r}_{i}\right)$, $\mathbf{H}_{i}^{0}=\mathbf{H}^{0}\left(\mathbf{r}_{i}\right)$, and

$$
\hat{G}_{i j}=A_{i j} \hat{I}+B_{i j}\left(\frac{\mathbf{r}_{i j} \otimes \mathbf{r}_{i j}}{r_{i j}^{2}}\right), \quad \hat{C}_{i j}=D_{i j} \frac{\mathbf{r}_{i j}}{r_{i j}} \times,
$$

where $\hat{I}$ is a $3 \times 3$ unit tensor, $\otimes$ denotes a tensor product, and $A_{i j}, B_{i j}$ and $D_{i j}$ are defined as follows:

$$
\begin{aligned}
A_{i j} & =\frac{\exp \left(i k r_{i j}\right)}{r_{i j}}\left(k^{2}-\frac{1}{r_{i j}^{2}}+\frac{i k}{r_{i j}}\right), \\
B_{i j} & =\frac{\exp \left(i k r_{i j}\right)}{r_{i j}}\left(-k^{2}+\frac{3}{r_{i j}^{2}}-\frac{3 i k}{r_{i j}}\right), \\
D_{i j} & =\frac{\exp \left(i k r_{i j}\right)}{r_{i j}}\left(k^{2}+\frac{i k}{r_{i j}}\right),
\end{aligned}
$$

where $r_{i j}=\left|\mathbf{r}_{i j}\right|=\left|\mathbf{r}_{i}-\mathbf{r}_{j}\right|$ is center-to-center distance between $i$-th and $j$-th particles, $k=2 \pi / \lambda$ is a wave number, and $\lambda$ is a wavelength of external illumination.

Electric and magnetic dipole polarizabilities are explicitly defined as [81]:

$$
\begin{aligned}
\alpha_{i}^{e} & =\frac{3 i}{2 k^{3}} \frac{n \psi_{1}\left(n k R_{i}\right) \psi_{1}^{\prime}\left(k R_{i}\right)-\psi_{1}\left(k R_{i}\right) \psi_{1}^{\prime}\left(n k R_{i}\right)}{n \psi_{1}\left(n k R_{i}\right) \xi_{1}^{\prime}\left(k R_{i}\right)-\xi_{1}\left(k R_{i}\right) \psi_{1}^{\prime}\left(n k R_{i}\right)}, \\
\alpha_{i}^{m} & =\frac{3 i}{2 k^{3}} \frac{\psi_{1}\left(n k R_{i}\right) \psi_{1}^{\prime}\left(k R_{i}\right)-n \psi_{1}\left(k R_{i}\right) \psi_{1}^{\prime}\left(n k R_{i}\right)}{\psi_{1}\left(n k R_{i}\right) \xi_{1}^{\prime}\left(k R_{i}\right)-n \xi_{1}\left(k R_{i}\right) \psi_{1}^{\prime}\left(n k R_{i}\right)},
\end{aligned}
$$

where $n$ is the refractive index of the NP material, $R_{i}$ is the radius of the $i$-th particle, $\psi_{1}(x)$ and $\xi_{1}(x)$ are Riccati-Bessel functions, and prime denotes the derivation with respect to the argument in parentheses.

The electric $\mathbf{d}_{i}$ and magnetic $\mathbf{m}_{i}$ dipoles induced on each NP can be found from the solution of Eq. (1). In this work, we describe the optical response of the NPs array with the extinction efficiency

$$
Q_{e}=\frac{4 k}{I_{0} N\langle R\rangle^{2}} \operatorname{Im} \sum_{i=1}^{N}\left(\mathbf{d}_{i} \cdot \mathbf{E}_{i}^{0 *}+\frac{\mu_{0}}{\varepsilon_{0}} \mathbf{m}_{i} \cdot \mathbf{H}_{j}^{0 *}\right),
$$

where $I_{0}$ is the intensity of the incident field, and the asterisk denotes a complex conjugate. Note that in the general case of polydisperse array with $R_{i} \neq R$, the mean radius $\langle R\rangle=$ $\sum_{i=1}^{N} R_{i} / N$ is used to define $Q_{e}$.

The coupled dipole approximation quite accurately describes optical properties of arrays from relatively small Si NPs. Fullwave simulations [82] show that ED and MD are predominant in arrays of Si NPs with $R=65 \mathrm{~nm}$, and high order electric and magnetic field oscillations can be ignored in this case. Though, higher-order multipoles in all-dielectric NPs are pronounced for example, in large [83-85] and nonspherical [86] single Si NPs, or closely packed arrays of Si NPs [40].

\section{ORDERED ARRAYS}

We start with the optical properties of a single Si nanosphere. Though it was numerously discussed in the literature, for instance, in Ref. [39], we plot these results for the Reader's convenience. Figure 1(a) shows the refractive index of $\mathrm{Si}$ used in calculations [87], while Fig. 1(b) shows the extinction efficiency $Q_{e}$ for a single $\mathrm{Si}$ nanosphere of various radii $R$. For a single sphere, and only in this case, we calculate $Q_{e}$ taking into account high-order harmonics [81] required for the convergence of the electromagnetic light scattering problem [88]. It can be seen from Fig. 1(b) that indeed, for given sizes, Si nanospheres have distinct and predominant ED and MD resonances in the visible wavelength range. In what follows, we consider arrays from $\mathrm{Si}$ nanospheres with $R=65 \mathrm{~nm}$ radius. However, in the special case of a size disorder, all possible radii of NPs will fall into the range shown in Fig. 1(b), i.e. $50 \mathrm{~nm} \leq R_{i} \leq 80 \mathrm{~nm}$. Therefore, the coupled dipole approximation can be used with a strong confidence.
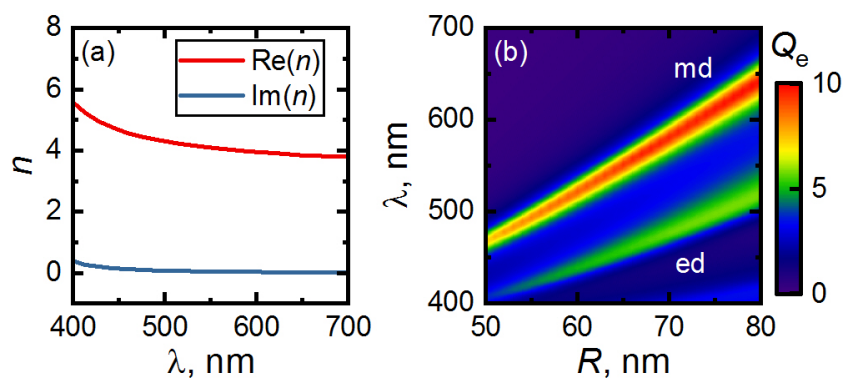

Fig. 1. (a) Refractive index $n$ of Si from Ref. [87]; (b) Extinction spectra for a single Si NP of various radii $R$ taking into account high-order multipoles. Spectral positions of electric and magnetic dipole resonances are denoted as 'ed' and 'md', respectively.

Next, it is insightful to discuss optical properties of ordered Si nanostructures. Figures 2(a) and 2(b) show two different types 

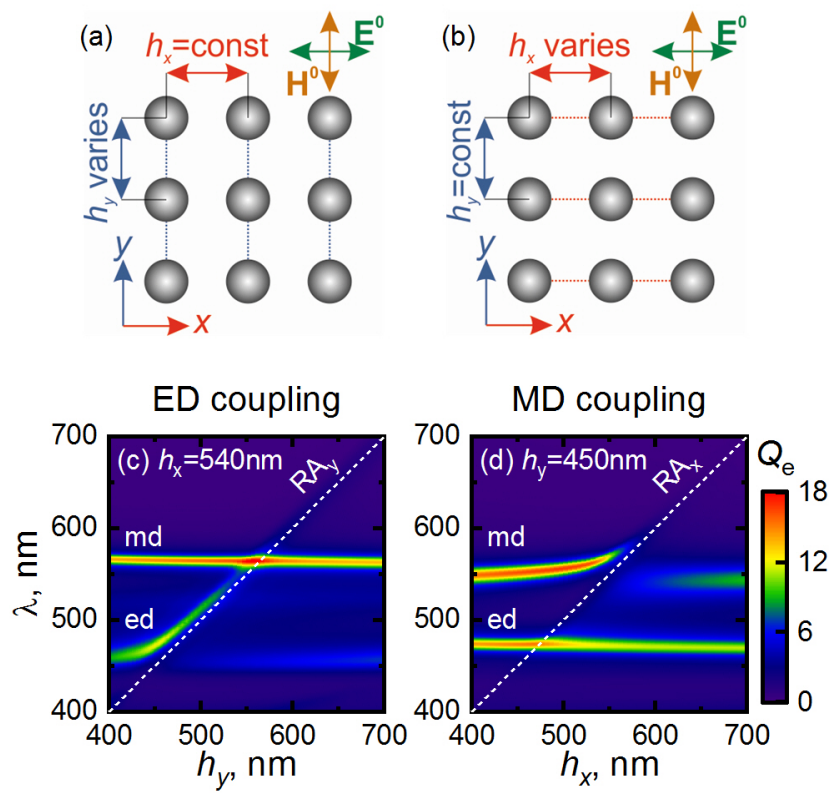

Fig. 2. Schematic representation (top rows) and extinction spectra $Q_{e}$ (bottom rows) of ordered $2 D$ lattices from $N=20 \times$ $20 \mathrm{Si}$ NPs with $R=65 \mathrm{~nm}$. Two configurations are considered: (left) fixed $h_{x}=540 \mathrm{~nm}$ and varying $h_{y}$, and (right) fixed $h_{y}=450 \mathrm{~nm}$ and varying $h_{x}$. Spectral positions of ED and MD resonances are denoted as 'ed' and 'md', respectively. Dashed $\mathrm{RA}_{x}$ and $\mathrm{RA}_{y}$ lines denote Rayleigh anomalies $\lambda=h_{x}$ and $\lambda=h_{y}$, correspondingly.

of lattices which have been studied in this work: (i) with fixed period along the $x$ axis, $h_{x}$, and varying period along the $y$ axis, $h_{y}$, and (ii) with fixed $h_{y}$ and varying $h_{x}$. Such variations of interparticle distances make it possible to get ED or MD coupling with lattice modes [55]. In both cases, the incident electric $\mathbf{E}^{0}$ and magnetic $\mathbf{H}^{0}$ fields are aligned along the $x$ and $y$ axes, correspondingly. Lattices from $N=20 \times 20$ Si NPs have been considered.

In the first case, as it is clearly seen from Fig. 2(c), ED strongly couples to lattice modes which leads to the emergence of quite sharp collective lattice resonances. The position of the MD resonance slightly shifts to shorter wavelengths for large $h_{y}$. Note that $Q_{e}$ for MD increases near the Rayleigh anomaly $\lambda=h_{y}$. In the second case, according to Fig. 2(d), the same strong coupling with lattice modes occurs for MD, while the position of ED gradually shifts to shorter wavelengths and the corresponding $Q_{e}$ decreases with increasing $h_{x}$. Thus, the coupling occurs for the incident field (electric or magnetic) perpendicular to the axis along which the interparticle distance is changed. In other words, for the particular case considered in this work, EDs $\left(\mathbf{E}^{0}\right.$ is parallel to $x$ axis) couple to $\mathrm{RA}_{y}$, and vice versa, $\mathrm{MDs}\left(\mathrm{H}^{0}\right.$ is parallel to $y$ axis) couple to $\mathrm{RA}_{x}$.

\section{DISORDERED ARRAYS}

\section{A. Types of disorder}

According to Eq. (1), two types of disorder can be distinguished [69]: (i) off-diagonal and (ii) diagonal. These types affect either off-diagonal or diagonal elements of the interaction matrix in Eq. (1), respectively. The first type of disorder affects only tensors $\hat{G}_{i j}$ and $\hat{C}_{i j}$ which are the functions of the NPs posi-

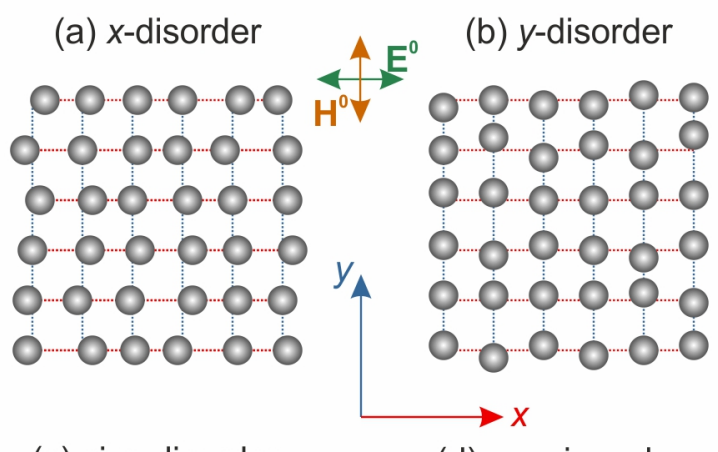

(c) size disorder

(d) quasi-random
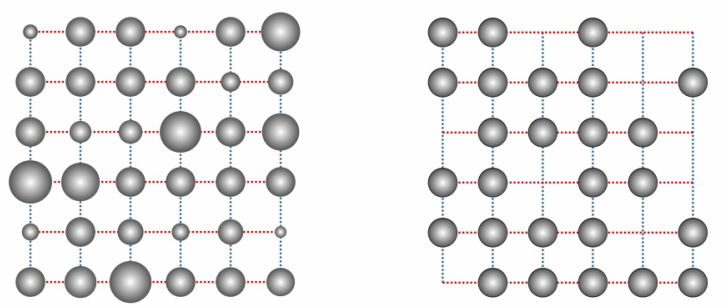

Fig. 3. Schematic representation of different types of disorder considered in this work: (a) $x$-disorder, (b) $y$-disorder, (c) size disorder, and (d) quasi-random array.

tions, while the second type of disorder affects only $\alpha_{i}^{e, m}$ which are the functions of NPs shape and size.

As it was shown for ordered arrays of NPs in Fig. 2, two types of coupling can be distinguished. For fixed illumination, optical response of lattices strongly depends on variations of either $h_{x}$ and $h_{y}$. Thus, to get more insights, we introduce the offdiagonal disorder in the following manner. We study positional disorder along the $x$ axis keeping $y$ coordinates constant, and vice versa, as shown in Fig. 3(a) and 3(b), respectively. We will refer to these two types of positional disorder as $x$-disorder and $y$-disorder, correspondingly. For both cases, we introduce deviations $\sigma_{x, y}$ which characterize a degree of disorder. For each $i$-th particle with initial $\left(x_{i}, y_{i}\right)$ coordinates, we randomly set new coordinates as $\left(x_{i}^{\text {dis }}, y_{i}\right)$ for $x$-disorder and $\left(x_{i}, y_{i}^{\text {dis }}\right)$ for $y$-disorder within the following limits:

$$
x_{i}-\sigma_{x} \leq x_{i}^{\mathrm{dis}} \leq x_{i}+\sigma_{x}, \quad \text { and } \quad y_{i}-\sigma_{y} \leq y_{i}^{\text {dis }} \leq y_{i}+\sigma_{y} .
$$

Both $x_{i}^{\text {dis }}$ and $y_{i}^{\text {dis }}$ are randomly generated using a uniform distribution for each $i$-th NP and for each lattice with given $\left(h_{x}, h_{y}\right)$. Thus, the effects of positional disorder are uncorrelated.

The schematics of the lattice with diagonal (size) disorder is shown in Fig. 3(c). In this case, we keep original coordinates of each NP, and randomly change the radius $R_{i}$ of each $i$-th NP within the following limits using a uniform distribution:

$$
R_{i}-\sigma_{R} \leq R_{i}^{\text {dis }} \leq R_{i}+\sigma_{R} .
$$

Again, as in the case of off-diagonal disorder, $R_{i}^{\text {dis }}$ is introduced randomly for each NP and for each lattice configuration, which provides uncorrelated results.

Finally, Fig. 3(d) shows a special combination of diagonal and off-diagonal disorders, which attracts specific interest [64, 65]. It is a well-known fact that the coupling between a single NP resonance and lattice modes strongly depends on the number of 

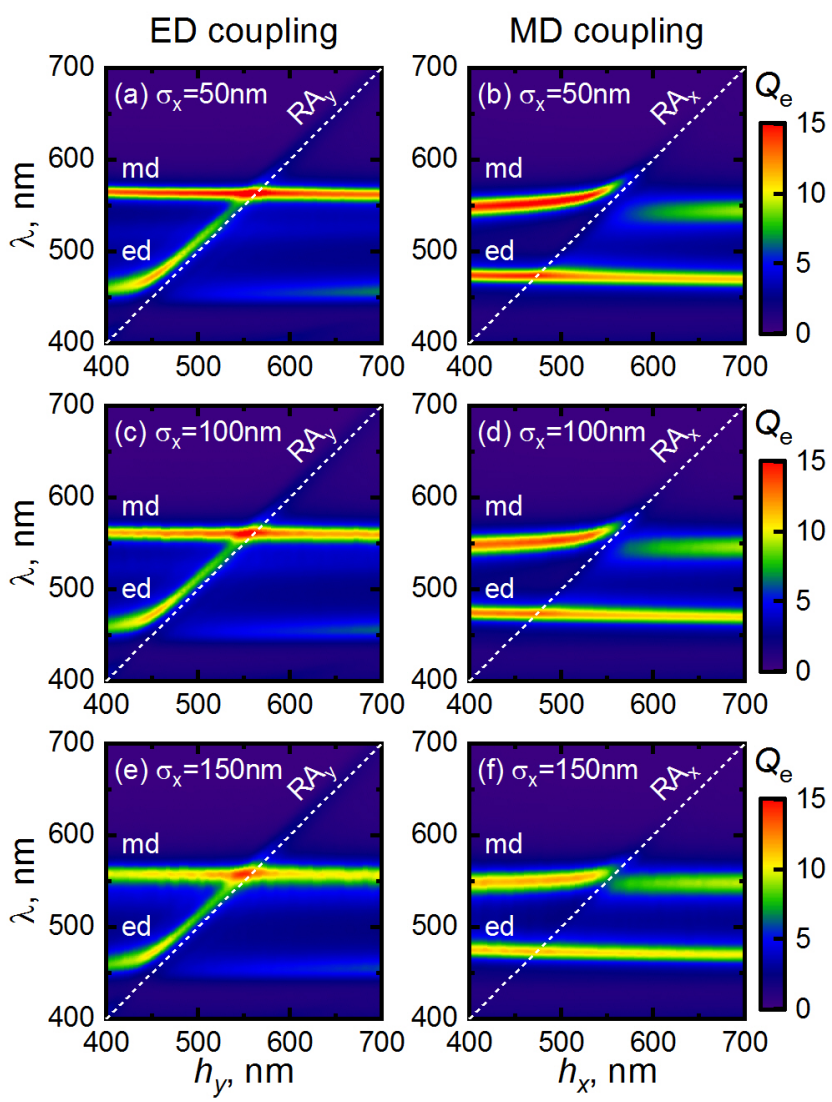

Fig. 4. Extinction spectra $Q_{e}$ for the same $2 D$ lattices as in Figs. 2(c) and 2(d), but for various degrees of positional disorder $\sigma_{x}$ along $x$ axis, as shown in Fig 3(a).

NPs in the array $[89,90]$. However, periodic lattices of strictly arranged NPs are usually considered to study this finite-size effect. In our work, we fix the initial coordinates and the sizes of NPs, and randomly remove NPs from the lattice, keeping other NPs untouched. This type of imperfections is somewhat similar to vacancies in crystal structures. In what follows, we will refer to lattices shown in Fig. 3(d) as to quasi-random arrays.

We emphasize that each lattice configuration for each type of disorder with given $\sigma_{x}, \sigma_{y}$ and $\sigma_{R}$ or number of NPs removed from the lattice in the case of quasi-random arrays, has been simulated only once, without computing ensemble averages. The reasonable closeness to statistical average has been granted by simulating large enough number of NPs.

\section{B. Off-diagonal (positional) disorder}

Figures 4 and 5 show extinction spectra for arrays of NPs with different degrees of $x$ - and $y$-disorders. It can be seen that these two types of positional disorder affect the optical properties of NPs in a different way, depending on the coupling regime.

As it might be expected from the analysis of Fig. 2(d), the $x$-disorder significantly affects $\mathrm{MD}$, since the latter strongly couples to the Rayleigh anomaly $\mathrm{RA}_{x}$. Clearly, from Fig. 4, one may observe slight suppression of the MD with the increasing of the degree of disorder, $\sigma_{x}$, both for ED and MD coupling scenarios. It also has to be noticed that the coupling of MD and $\mathrm{RA}_{x}$ remains observable even for sufficiently large $\sigma_{x}$ in Fig. 4(f), where $\mathrm{MD}$ is suppressed. The ED remains almost the same for each case shown in Fig. 4.
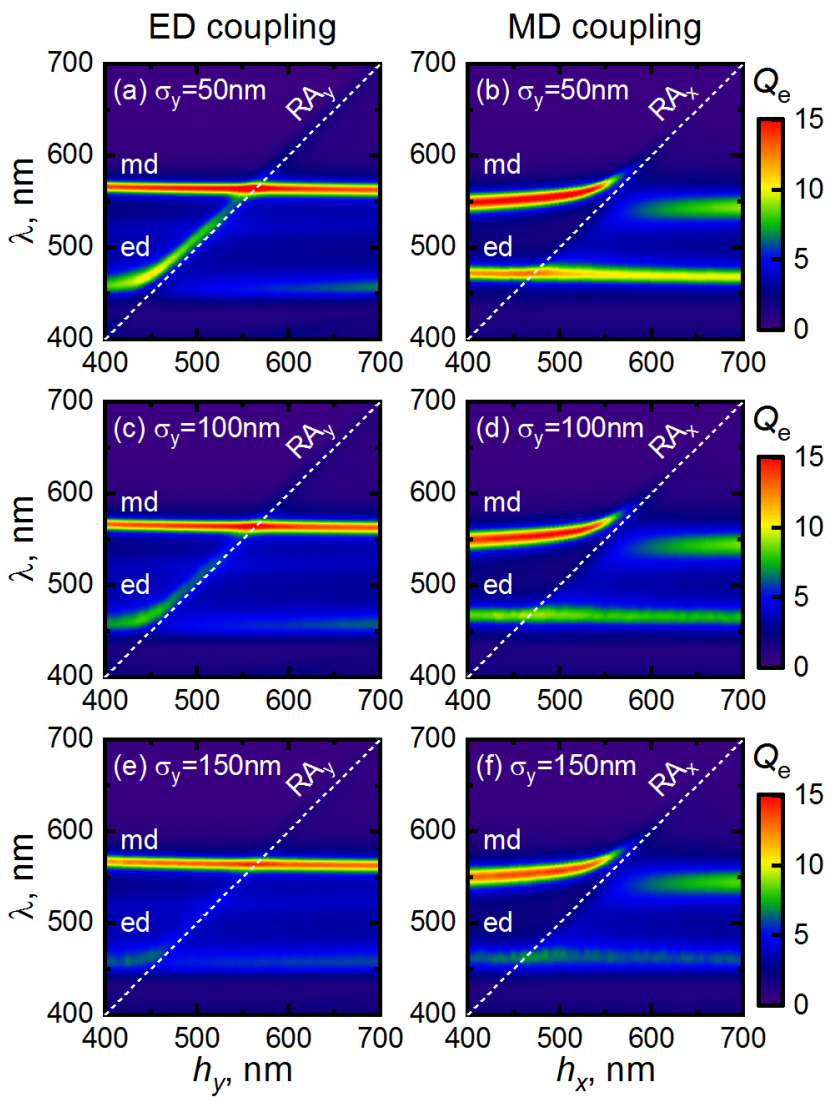

Fig. 5. Same as in Fig. 4, but for various degrees of positional disorder $\sigma_{y}$ along $y$ axis, as shown in Fig 3(b).

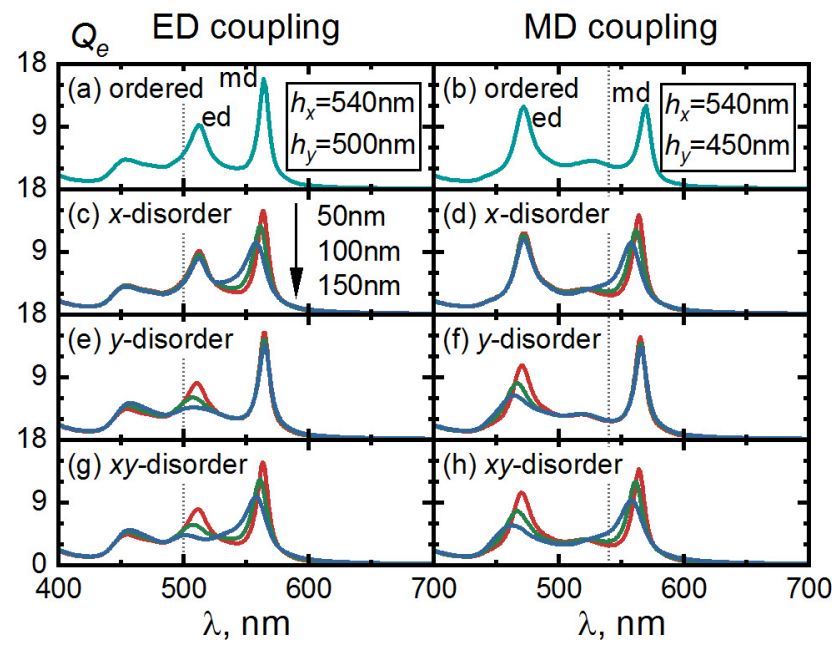

Fig. 6. Extinction spectra $Q_{e}$ of NPs arrays with ED coupling (left), and MD coupling (right) for various degrees of positional disorder $\sigma_{x}(\mathrm{c}, \mathrm{d}), \sigma_{y}(\mathrm{e}, \mathrm{f})$, and $\sigma_{x y}(\mathrm{~g}, \mathrm{~h})$. Corresponding values of $h_{x}$ and $h_{y}$ are shown in legends. Dashed vertical lines denote positions of Rayleigh anomalies $\mathrm{RA}_{y}$ at $\lambda=500 \mathrm{~nm}$ (left), and $\mathrm{RA}_{x}$ at $\lambda=540 \mathrm{~nm}$ (right).

Figure 5 shows an expected trend: since ED couples to $\mathrm{RA}_{y}$, $y$-disorder affects only the former, keeping MD almost the same for various $\sigma_{y}$. However, Figs. 5(e)-5(f) show almost total suppression of ED for $\sigma_{y}=150 \mathrm{~nm}$, while in the case of strong 

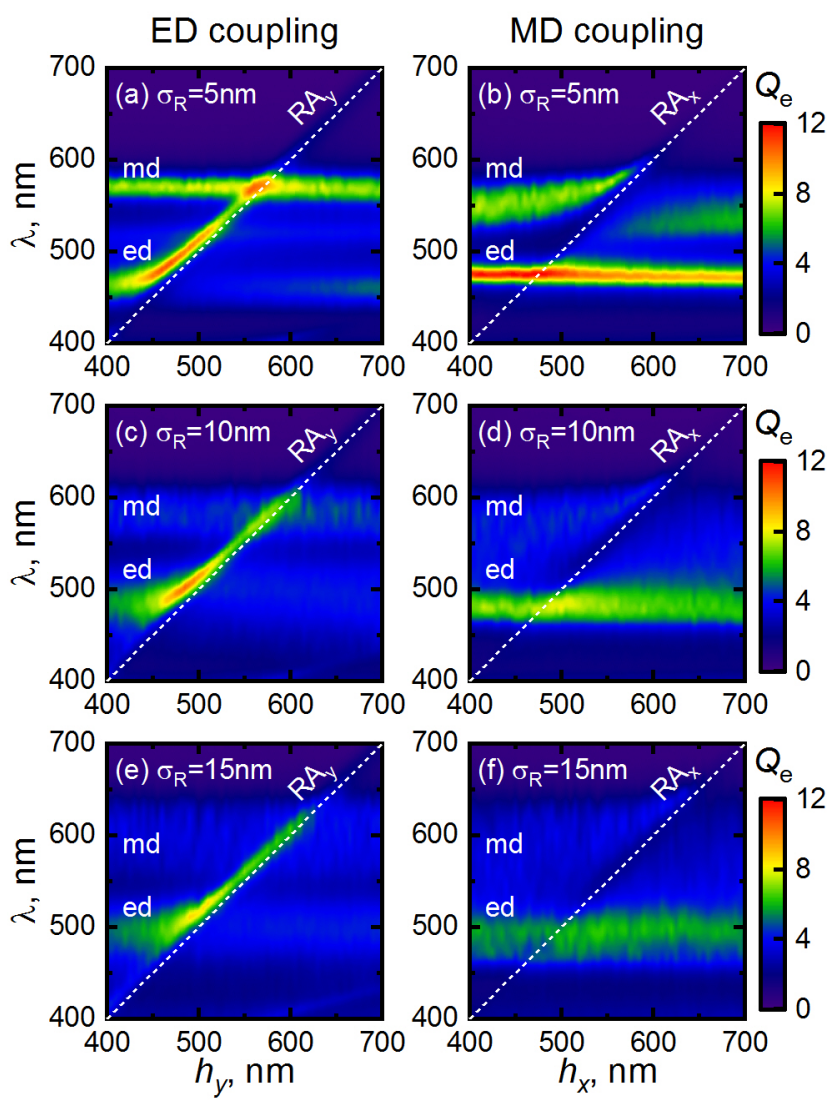

Fig. 7. Extinction spectra $Q_{e}$ for the same $2 D$ lattices as in Figs. 2(c) and 2(d), but for various degrees of size disorder $\sigma_{R}$, as shown in Fig 3(c).

$x$-disorder shown in Figs. 4(e)-4(f), MD is quite pronounced.

Finally, Fig. 6 shows the detailed comparison of the extinction spectra for arrays with ED or MD couplings. Indeed, $x$-disorder strongly suppresses the MD, while $y$-disorder suppresses the ED resonance. Since the ED is generally weaker than the MD, the former is almost completely disappears for high degrees of $y$-disorder. For the completeness, Figs. 6(g)-6(h) show the spectra for arrays with $x y$-disorder, which has been introduced in the same way as the $x$-and $y$-disorders, but with simultaneous randomization of both $x_{i}$ and $y_{i}$ coordinates of each NP. It can be seen that in general, such a combined disorder yield in a superposition of both $x$ - and $y$-disorders which suppresses both ED and MD resonances.

\section{Diagonal (size) disorder}

Figure 7 shows extinction spectra for arrays with various degrees of size disorder, $\sigma_{R}$. It is clearly seen that random variations of NP sizes strongly suppress both ED and MD resonances. However, MD remains observable only for $\sigma_{R}=5 \mathrm{~nm}$, while for larger $\sigma_{R}$ it almost completely disappears. Contrary, the ED resonance is preserved in all cases, and, of note, EDs strongly couple with Rayleigh anomalies, $\mathrm{RA}_{y}$, even for high degrees of diagonal disorder, as shown in Fig. 7(e). This effect might be explained by different behavior of polarizabilities $\alpha_{i}^{e}$ and $\alpha_{i}^{m}$ [39] which yields in different impact of size disorder on ED and MD resonances.

To get a deeper insight, we plot $Q_{e}$ for arrays with fixed $h_{x}$ and $h_{y}$, as shown in Fig. 8. Indeed, Figs. 8(c), 8(e), 8(g) show that

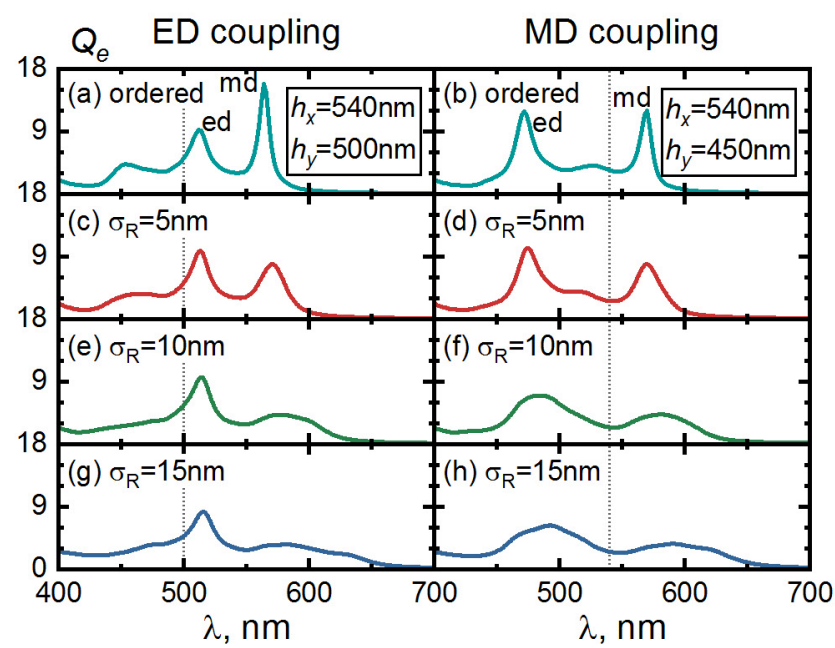

Fig. 8. Extinction spectra $Q_{e}$ of NPs arrays with ED coupling (left), and MD coupling (right) for various degrees of size disorder $\sigma_{R}$. Corresponding values of $h_{x}$ and $h_{y}$ are shown in legends. Dashed vertical lines denote positions of Rayleigh anomalies $\mathrm{RA}_{y}$ at $\lambda=500 \mathrm{~nm}$ (left), and $\mathrm{RA}_{x}$ at $\lambda=540 \mathrm{~nm}$ (right).

size disorder has a surprisingly weak effect on the ED resonance of arrays with strong ED coupling. It can be seen from Fig. 8(g) that maximum $Q_{e}$ for the ED resonance drops by no more than $10 \%$ for $\sigma_{R}=15 \mathrm{~nm}$ compared to the ordered array shown in Fig. 8(a). For arrays with MD coupling, $Q_{e}$ for ED resonance drops stronger, by the factor of 2 for $\sigma_{R}=15 \mathrm{~nm}$, as shown in Fig. 8(h). As for the MD resonance, in both the ED and MD coupling cases, the extinction efficiency for MD sharply drops for $\sigma_{R}=5 \mathrm{~nm}$. For larger $\sigma_{R}$, the MD resonance becomes almost indistinguishable.

\section{Quasi-random arrays}

From the previous discussion of diagonal and off-diagonal types of disorder, we can conclude that simultaneous implementation of positional and size disorders should likely result in the superposition of the effects shown in Figs. 4, 5 and 7. Thus, we do not consider arrays of randomly located NPs of different size. Instead, we introduce a specific combination of positional and size disorders as shown in Fig. 3(d). These quasi-random arrays are fundamentally different from ones shown in Figs. 3(a)-3(c) since random elements of the interaction matrix in Eq. (1) are strictly set to zero in the case of quasi-random arrays, while in previously considered scenarios, off-diagonal or diagonal elements have acquired random deviations according to $\sigma_{x}, \sigma_{y}$ or $\sigma_{R}$

Here, we consider NPs with the same size, $R=65 \mathrm{~nm}$, but increase their number to $N=30 \times 30$ (while previously discussed arrays had $N=20 \times 20 \mathrm{NPs}$ ). Next, we randomly remove 171 , 459 or 756 NPs, leaving the rest $81 \%, 49 \%$ or $16 \%$ of NPs untouched, respectively. We note that the consideration of larger arrays is preferable for this type of disorder, since coupling effects may be totally suppressed in arrays from small number of NPs left in the lattice [89]. However, in the smallest array considered here, we keep 144 quasi-randomly located NPs, which is sufficient for the emergence of coupling effects.

Intuitively, one could expect the suppression of ED and MD couplings with the increasing number of NPs removed from 

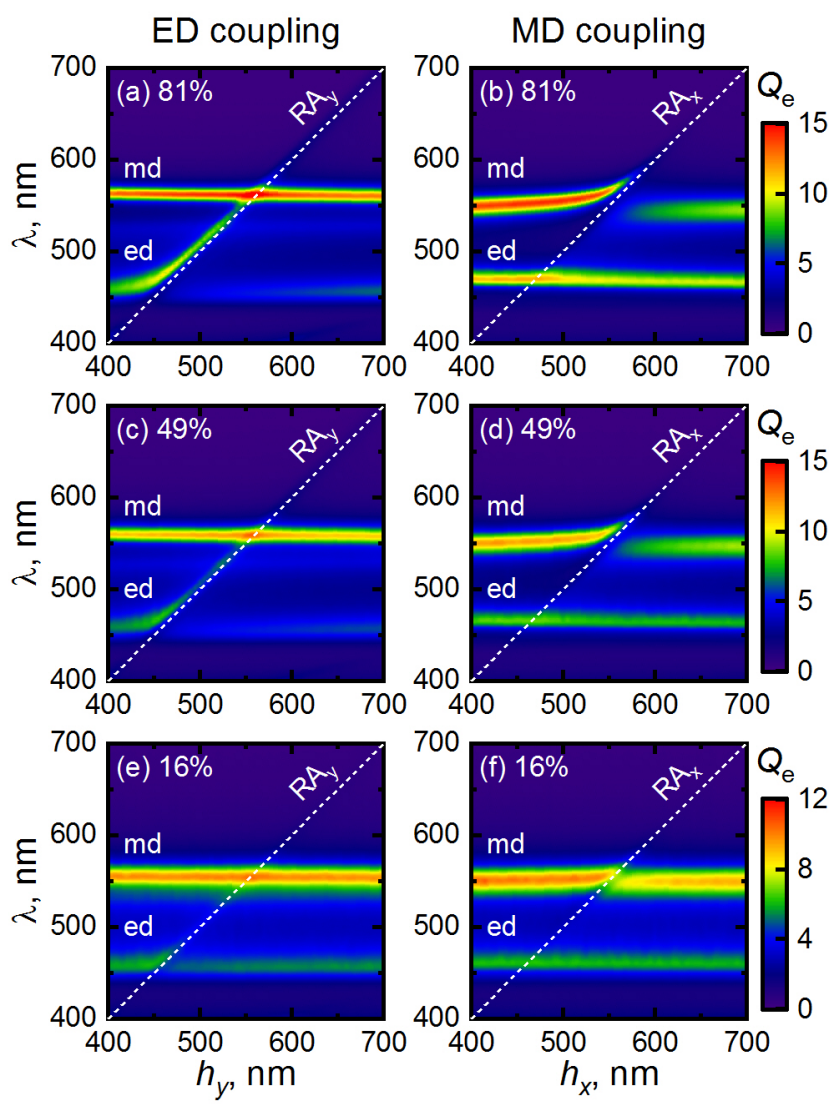

Fig. 9. Extinction spectra $Q_{e}$ for quasi-random $2 D$ lattices, as shown in Fig 3(d), for different number of NPs: (a)-(b) $81 \%=$ 729 , (c)-(d) $49 \%=441$, and (e)-(f) $16 \%=144$ kept untouched in $N=30 \times 30$ arrays of NPs with $R=65 \mathrm{~nm}$. Note the different color scale in the last row (e)-(f).

the ordered array. Indeed, Fig. 9 confirms such an expectation. However, it can be seen that lattices which contains $81 \%$ of the initial NPs have almost the same optical properties as the original periodic arrays. Moreover, Figs. 9(e)-9(f) show that ED and MD are coupled to Rayleigh anomalies (though quite weakly) in the arrays with only $16 \%$ NPs left, and extinction spectra of such arrays tend to become closer to $Q_{e}$ of a single NP.

For comparison, Fig.10 shows spectra of ordered arrays (as in Figs. 2(a)-2(b)) from exactly the same number of NPs as in quasi-random arrays, i.e. $27 \times 27,21 \times 21$, and $12 \times 12$, and with the same $h_{x}$ and $h_{y}$. It can be seen from 10(c)-10(d) that $Q_{e}$ of the quasi-random array from $729 \mathrm{NPs}$ is also almost the same as $Q_{e}$ for the periodic $27 \times 27$ array. Moreover, even with the increasing number of NPs removed from the array, $Q_{e}$ of the quasi-random lattices is quite close to strictly ordered arrays with the same number of NPs. However, in the most extreme cases of quasi-random arrays shown in Fig. 10(g)-10(h), the collective ED resonances are almost suppressed, while the MD coupling remains observable, though, the corresponding peak of MD resonance is blue-shifted compared with the ordered arrays.

\section{CONCLUSION}

To conclude, we have theoretically analyzed the impact of various types of imperfections on the optical response of $2 D$ arrays of spherical Si nanoparticles. Electric and magnetic dipole resonances are dominant in Si nanospheres in the considered

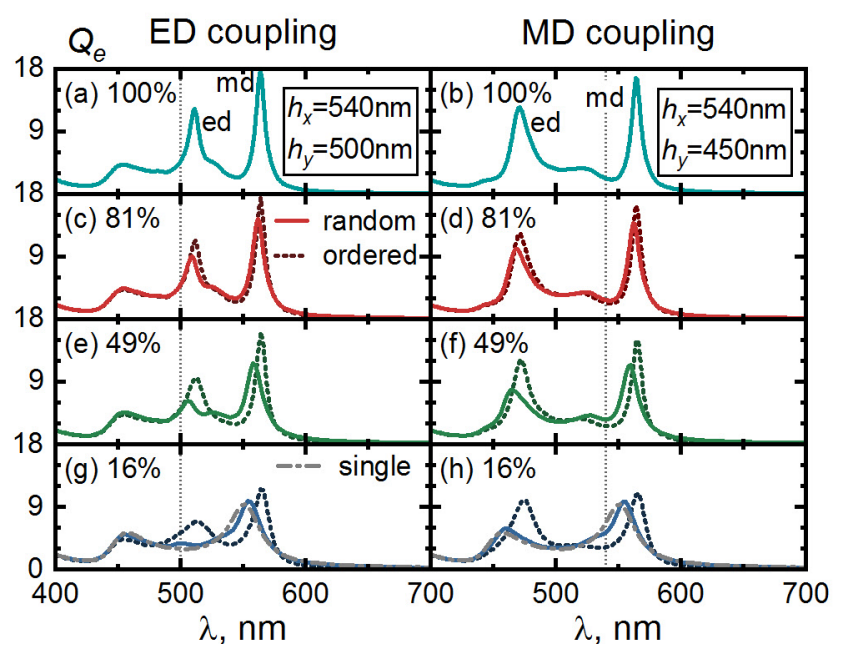

Fig. 10. Extinction spectra $Q_{e}$ of NPs arrays with ED coupling (left), and MD coupling (right) for (a)-(b) $N=30 \times 30$ array, and for its various quasi-random modifications (solid lines): (c)-(d) $81 \%=729$, (e)-(f) $49 \%=441$, and (g)-(h) $16 \%=$ 144 NPs kept untouched. For comparison, $Q_{e}$ of strictly periodic (dashed lines) arrays of the same number of NPs are shown: (c)-(d) $N=27 \times 27=729$, (e)-(f) $N=21 \times 21=441$, and (g)-(h) $N=12 \times 12=144$, grey dash-dot lines show $Q_{e}$ of a single Si NP with $R=65 \mathrm{~nm}$. Corresponding values of $h_{x}$ and $h_{y}$ are shown in legends. Dashed vertical lines denote positions of Rayleigh anomalies $\mathrm{RA}_{y}$ at $\lambda=500 \mathrm{~nm}$ (left), and $\mathrm{RA}_{x}$ at $\lambda=540 \mathrm{~nm}$ (right).

range $50 \mathrm{~nm} \leq R \leq 80 \mathrm{~nm}$, thus, we have used the coupled dipole approximation which adequately describes electromagnetic properties of Si nanoparticle arrays [82].

We first have shown the existence of two types of collective resonances in $2 D$ arrays emerging from the strong coupling of either electric or magnetic dipole resonances of a single NP with lattice modes (Rayleigh anomalies) of the $2 D$ array. Such a coupling occurs when the corresponding component of the incident field (electric or magnetic) is orthogonal to the varied period $\left(h_{y}\right.$ or $\left.h_{x}\right)$ of the lattice, while the other period $\left(h_{x}\right.$ or $\left.h_{y}\right)$ is constant [55].

Second, we have shown that electric or magnetic responses are affected by the positional disorder only when nanoparticles are shifted along the axis which is orthogonal to the corresponding component of incident electromagnetic illumination. In our case, for $\mathbf{E}^{0} \| x$ and $\mathbf{H}^{0} \| y$, electric and magnetic dipole resonances are strongly suppressed only for $y$ - or $x$-disorders, respectively. Obviously, both resonances are affected when nanoparticles are shifted along the $x$ and $y$ axes simultaneously.

Next, we have demonstrated that collective magnetic dipole response almost completely vanishes in the case of diagonal (size) disorder with $\sigma_{R}>5 \mathrm{~nm}$. However, the electric counterpart remains quite stable, especially in the case of strong collective coupling between the electric dipole resonance and lattice modes, even for highly polydisperse arrays with $\sigma_{R}=15 \mathrm{~nm}$.

Finally, we have considered quasi-random arrays as a special combination of off-diagonal and diagonal disorders. Instead of simultaneously shifting nanoparticles and changing their sizes, we have randomly removed nanoparticles from the lattice keeping other nanoparticles at original points with original sizes. Surprisingly, arrays with only $16 \%$ nanoparticles left in the lat- 
tice exhibit both electric and magnetic collective resonances. However, the extinction spectra of such arrays tend to be similar to spectra of a single nanoparticle.

Reported results provide a comprehensive analysis and a fundamental understanding of the impact that disorder has on collective resonances in $2 D$ arrays of all-dielectric nanoparticles. While we have considered spherical Si nanoparticles embedded in vacuum, one could expect the similar trends for all-dielectric arrays of nanoparticles of other shapes or materials [91], as long as high-order multipoles can be neglected. Thus, we believe that reported results may pave the way to future applications in all-dielectric nanophotonics.

\section{ACKNOWLEDGMENT}

The reported study was funded by Russian Foundation for Basic Research, Government of Krasnoyarsk Territory, Krasnoyarsk Regional Fund of Science, the research project No 18-42-240013; the State contract with Siberian Federal University for scientific research in 2017-2019 (Grant No.3.8896.2017). H.A. and V.Z. acknowledge the support of the Russian Science Foundation (Project No.18-13-00363) (numerical calculations of spectral properties of planar dielectric nanostructures).

\section{REFERENCES}

1. S. Zou and G. C. Schatz, "Narrow plasmonic/photonic extinction and scattering line shapes for one and two dimensional silver nanoparticle arrays," J. Chem. Phys. 121, 12606-12612 (2004).

2. S. Zou, N. Janel, and G. C. Schatz, "Silver nanoparticle array structures that produce remarkably narrow plasmon lineshapes," J. Chem. Phys. 120, 10871-10875 (2004).

3. V. A. Markel, "Divergence of dipole sums and the nature of nonLorentzian exponentially narrow resonances in one-dimensional periodic arrays of nanospheres," J. Phys. B: At. Mol. Opt. Phys. 38, L115-L121 (2005).

4. B. Auguié and W. L. Barnes, "Collective Resonances in Gold Nanoparticle Arrays," Phys. Rev. Lett. 101, 143902 (2008).

5. Y. Chu, E. Schonbrun, T. Yang, and K. B. Crozier, "Experimental observation of narrow surface plasmon resonances in gold nanoparticle arrays," Appl. Phys. Lett. 93, 181108 (2008).

6. V. G. Kravets, F. Schedin, and A. N. Grigorenko, "Extremely Narrow Plasmon Resonances Based on Diffraction Coupling of Localized Plasmons in Arrays of Metallic Nanoparticles," Phys. Rev. Lett. 101, 087403 (2008).

7. F. van Beijnum, P. J. van Veldhoven, E. J. Geluk, M. J. A. de Dood, G. W. 't Hooft, and M. P. van Exter, "Surface Plasmon Lasing Observed in Metal Hole Arrays," Phys. Rev. Lett. 110, 206802 (2013).

8. W. Zhou, M. Dridi, J. Y. Suh, C. H. Kim, D. T. Co, M. R. Wasielewski, G. C. Schatz, and T. W. Odom, "Lasing action in strongly coupled plasmonic nanocavity arrays," Nat. Nanotechnol. 8, 506-511 (2013).

9. M. Dridi and G. C. Schatz, "Model for describing plasmon-enhanced lasers that combines rate equations with finite-difference time-domain," J. Opt. Soc. Am. B 30, 2791 (2013).

10. A. H. Schokker and A. F. Koenderink, "Lasing at the band edges of plasmonic lattices," Phys. Rev. B 90, 155452 (2014).

11. D. Wang, W. Wang, M. P. Knudson, G. C. Schatz, and T. W. Odom, "Structural Engineering in Plasmon Nanolasers," Chem. Rev. 118, 2865-2881 (2018).

12. A. H. Schokker, F. van Riggelen, Y. Hadad, A. Alù, and A. F. Koenderink, "Systematic study of the hybrid plasmonic-photonic band structure underlying lasing action of diffractive plasmon particle lattices," Phys. Rev. B 95, 085409 (2017).

13. R. Adato, A. A. Yanik, J. J. Amsden, D. L. Kaplan, F. G. Omenetto, M. K. Hong, S. Erramilli, and H. Altug, "Ultra-sensitive vibrational spectroscopy of protein monolayers with plasmonic nanoantenna arrays," Proc. Natl. Acad. Sci. 106, 19227-19232 (2009).
14. V. G. Kravets, F. Schedin, A. V. Kabashin, and A. N. Grigorenko, "Sensitivity of collective plasmon modes of gold nanoresonators to local environment," Opt. Lett. 35, 956 (2010).

15. R. Adato and H. Altug, "In-situ ultra-sensitive infrared absorption spectroscopy of biomolecule interactions in real time with plasmonic nanoantennas," Nat. Commun. 4 (2013).

16. B. D. Thackray, V. G. Kravets, F. Schedin, G. Auton, P. A. Thomas, and A. N. Grigorenko, "Narrow Collective Plasmon Resonances in Nanostructure Arrays Observed at Normal Light Incidence for Simplified Sensing in Asymmetric Air and Water Environments," ACS Photonics 1, 1116-1126 (2014).

17. A. Danilov, G. Tselikov, F. Wu, V. G. Kravets, I. Ozerov, F. Bedu, A. N. Grigorenko, and A. V. Kabashin, "Ultra-narrow surface lattice resonances in plasmonic metamaterial arrays for biosensing applications," Biosens. Bioelectron. 104, 102-112 (2018).

18. R. R. Gutha, S. M. Sadeghi, A. Hatef, C. Sharp, and Y. Lin, "Ultrahigh refractive index sensitivity via lattice-induced meta-dipole modes in flat metallic nanoantenna arrays," Appl. Phys. Lett. 112, 223102 (2018).

19. G. Vecchi, V. Giannini, and J. Gómez Rivas, "Shaping the Fluorescent Emission by Lattice Resonances in Plasmonic Crystals of Nanoantennas," Phys. Rev. Lett. 102, 146807 (2009).

20. M. Ramezani, G. Lozano, M. A. Verschuuren, and J. Gómez-Rivas, "Modified emission of extended light emitting layers by selective coupling to collective lattice resonances," Phys. Rev. B 94, 125406 (2016).

21. F. Laux, N. Bonod, and D. Gérard, "Single Emitter Fluorescence Enhancement with Surface Lattice Resonances," The J. Phys. Chem. C 121, 13280-13289 (2017).

22. R. Kamakura, S. Murai, K. Fujita, and K. Tanaka, "Enhanced Photoluminescence from Organic Dyes Coupled to Periodic Array of Zirconium Nitride Nanoparticles," ACS Photonics 5, 3057-3063 (2018).

23. A. S. Roberts, A. Pors, O. Albrektsen, and S. I. Bozhevolnyi, "Subwavelength plasmonic color printing protected for ambient use," Nano Lett. 14, 783-787 (2014).

24. X. Duan, S. Kamin, and N. Liu, "Dynamic plasmonic colour display," Nat. Commun. 8, 14606 (2017).

25. H. Wang, X. Wang, C. Yan, H. Zhao, J. Zhang, C. Santschi, and O. J. F. Martin, "Full Color Generation Using Silver Tandem Nanodisks," ACS Nano 11, 4419-4427 (2017).

26. H. Bertin, Y. Brûlé, G. Magno, T. Lopez, P. Gogol, L. Pradere, B. Gralak, D. Barat, G. Demésy, and B. Dagens, "Correlated Disordered Plasmonic Nanostructures Arrays for Augmented Reality," ACS Photonics 5, 2661-2668 (2018).

27. R. W. Wood, "On a Remarkable Case of Uneven Distribution of Light in a Diffraction Grating Spectrum," Proc. Phys. Soc. Lond. 18, 269-275 (1902).

28. L. Rayleigh, "On the Dynamical Theory of Gratings," Proc. Royal Soc. A: Math. Phys. Eng. Sci. 79, 399-416 (1907).

29. S.-Q. Li, P. Guo, D. B. Buchholz, W. Zhou, Y. Hua, T. W. Odom, J. B. Ketterson, L. E. Ocola, K. Sakoda, and R. P. H. Chang, "Plasmonic-Photonic Mode Coupling in Indium-Tin-Oxide Nanorod Arrays," ACS Photonics 1, 163-172 (2014).

30. A. Yang, A. J. Hryn, M. R. Bourgeois, W.-K. Lee, J. Hu, G. C. Schatz, and T. W. Odom, "Programmable and reversible plasmon mode engineering," Proc. Natl. Acad. Sci. 113, 14201-14206 (2016).

31. D. Khlopin, F. Laux, W. P. Wardley, J. Martin, G. A. Wurtz, J. Plain, N. Bonod, A. V. Zayats, W. Dickson, and D. Gérard, "Lattice modes and plasmonic linewidth engineering in gold and aluminum nanoparticle arrays," J. Opt. Soc. Am. B 34, 691 (2017).

32. M. L. Tseng, J. Yang, M. Semmlinger, C. Zhang, P. Nordlander, and N. J. Halas, "Two-Dimensional Active Tuning of an Aluminum Plasmonic Array for Full-Spectrum Response," Nano Lett. 17, 6034-6039 (2017).

33. Y. Kawachiya, S. Murai, M. Saito, H. Sakamoto, K. Fujita, and K. Tanaka, "Collective plasmonic modes excited in Al nanocylinder arrays in the UV spectral region," Opt. Express 26, 5970 (2018).

34. R. Kamakura, S. Murai, S. Ishii, T. Nagao, K. Fujita, and K. Tanaka, "Plasmonic-Photonic Hybrid Modes Excited on a Titanium Nitride Nanoparticle Array in the Visible Region," ACS Photonics 4, 815-822 (2017). 
35. V. I. Zakomirnyi, I. L. Rasskazov, V. S. Gerasimov, A. E. Ershov, S. P. Polyutov, and S. V. Karpov, "Refractory titanium nitride two-dimensional structures with extremely narrow surface lattice resonances at telecommunication wavelengths," Appl. Phys. Lett. 111, 123107 (2017).

36. M. Kataja, T. K. Hakala, A. Julku, M. J. Huttunen, S. van Dijken, and P. Törmä, "Surface lattice resonances and magneto-optical response in magnetic nanoparticle arrays," Nat. Commun. 6, 7072 (2015).

37. M. Kataja, S. Pourjamal, N. Maccaferri, P. Vavassori, T. K. Hakala, M. J. Huttunen, P. Törmä, and S. van Dijken, "Hybrid plasmonic lattices with tunable magneto-optical activity," Opt. Express 24, 3652 (2016).

38. A. Christofi, Y. Kawaguchi, A. Alù, and A. B. Khanikaev, "Giant enhancement of Faraday rotation due to electromagnetically induced transparency in all-dielectric magneto-optical metasurfaces," Opt. Lett. 43, 1838 (2018).

39. A. B. Evlyukhin, C. Reinhardt, A. Seidel, B. S. Luk'Yanchuk, and B. N. Chichkov, "Optical response features of Si-nanoparticle arrays," Phys. Rev. B - Condens. Matter Mater. Phys. 82, 1-12 (2010).

40. R. S. Savelev, A. P. Slobozhanyuk, A. E. Miroshnichenko, Y. S. Kivshar, and P. A. Belov, "Subwavelength waveguides composed of dielectric nanoparticles," Phys. Rev. B 89, 035435 (2014).

41. E. N. Bulgakov and D. N. Maksimov, "Light guiding above the light line in arrays of dielectric nanospheres," Opt. Lett. 41, 3888 (2016).

42. R. M. Bakker, Y. F. Yu, R. Paniagua-Domínguez, B. Luk'yanchuk, and A. I. Kuznetsov, "Resonant Light Guiding Along a Chain of Silicon Nanoparticles," Nano Lett. 17, 3458-3464 (2017).

43. Q. Zhao, J. Zhou, F. Zhang, and D. Lippens, "Mie resonance-based dielectric metamaterials," Mater. Today 12, 60-69 (2009).

44. A. Arbabi, Y. Horie, M. Bagheri, and A. Faraon, "Dielectric metasurfaces for complete control of phase and polarization with subwavelength spatial resolution and high transmission," Nat. Nanotechnol. 10, 937943 (2015).

45. M. I. Shalaev, J. Sun, A. Tsukernik, A. Pandey, K. Nikolskiy, and N. M. Litchinitser, "High-Efficiency All-Dielectric Metasurfaces for Ultracompact Beam Manipulation in Transmission Mode," Nano Lett. 15, 6261-6266 (2015).

46. V. E. Babicheva, M. I. Petrov, K. V. Baryshnikova, and P. A. Belov, "Reflection compensation mediated by electric and magnetic resonances of all-dielectric metasurfaces [Invited]," J. Opt. Soc. Am. B 34, D18 (2017).

47. M. G. Barsukova, A. S. Shorokhov, A. I. Musorin, D. N. Neshev, Y. S. Kivshar, and A. A. Fedyanin, "Magneto-Optical Response Enhanced by Mie Resonances in Nanoantennas," ACS Photonics 4, 2390-2395 (2017).

48. V. E. Babicheva and A. B. Evlyukhin, "Resonant suppression of light transmission in high-refractive-index nanoparticle metasurfaces," Opt. Lett. 43, 5186 (2018).

49. F. Shen, Q. Kang, J. Wang, K. Guo, Q. Zhou, and Z. Guo, "Dielectric Metasurface-Based High-Efficiency Mid-Infrared Optical Filter," Nanomaterials. 8, 938 (2018).

50. S. Kruk and Y. Kivshar, "Functional Meta-Optics and Nanophotonics Governed by Mie Resonances," ACS Photonics 4, 2638-2649 (2017).

51. S. Tsoi, F. J. Bezares, A. Giles, J. P. Long, O. J. Glembocki, J. D. Caldwell, and J. Owrutsky, "Experimental demonstration of the optical lattice resonance in arrays of Si nanoresonators," Appl. Phys. Lett. 108, 111101 (2016).

52. L. Zhang, C. Ge, K. Zhang, C. Tian, X. Fang, W. Zhai, L. Tao, Y. Li, and G. Ran, "Lattice plasmons in dielectric nanoparticle arrays arranged on metal film," J. Opt. 18, 125002 (2016).

53. C.-Y. Yang, J.-H. Yang, Z.-Y. Yang, Z.-X. Zhou, M.-G. Sun, V. E. Babicheva, and K.-P. Chen, "Nonradiating Silicon Nanoantenna Metasurfaces as Narrowband Absorbers," ACS Photonics 5, 2596-2601 (2018).

54. D. Tzarouchis and A. Sihvola, "Light Scattering by a Dielectric Sphere: Perspectives on the Mie Resonances," Appl. Sci. 8, 184 (2018).

55. J. Li, N. Verellen, and P. Van Dorpe, "Engineering electric and magnetic dipole coupling in arrays of dielectric nanoparticles," J. Appl. Phys. 123, 083101 (2018).

56. V. E. Babicheva and J. V. Moloney, "Lattice effect influence on the elec- tric and magnetic dipole resonance overlap in a disk array," Nanophotonics. 7, 1663-1668 (2018).

57. M. B. Ross, C. A. Mirkin, and G. C. Schatz, "Optical Properties of OneTwo-, and Three-Dimensional Arrays of Plasmonic Nanostructures," The J. Phys. Chem. C 120, 816-830 (2016).

58. B. B. Rajeeva, L. Lin, and Y. Zheng, "Design and applications of lattice plasmon resonances," Nano Res. 11, 4423-4440 (2018).

59. W. Wang, M. Ramezani, A. I. Väkeväinen, P. Törmä, J. G. Rivas, and T. W. Odom, "The rich photonic world of plasmonic nanoparticle arrays," Mater. Today 21, 303-314 (2018).

60. V. G. Kravets, A. V. Kabashin, W. L. Barnes, and A. N. Grigorenko, "Plasmonic Surface Lattice Resonances: A Review of Properties and Applications," Chem. Rev. 118, 5912-5951 (2018).

61. B. Auguié and W. L. Barnes, "Diffractive coupling in gold nanoparticle arrays and the effect of disorder," Opt. Lett. 34, 401 (2009).

62. Y. Nishijima, L. Rosa, and S. Juodkazis, "Surface plasmon resonances in periodic and random patterns of gold nano-disks for broadband light harvesting," Opt. Express 20, 11466 (2012).

63. M. B. Ross, J. C. Ku, M. G. Blaber, C. A. Mirkin, and G. C. Schatz, "Defect tolerance and the effect of structural inhomogeneity in plasmonic DNA-nanoparticle superlattices," Proc. Natl. Acad. Sci. 112, 10292-10297 (2015).

64. A. H. Schokker and A. F. Koenderink, "Statistics of Randomized Plasmonic Lattice Lasers," ACS Photonics 2, 1289-1297 (2015).

65. A. H. Schokker and A. F. Koenderink, "Lasing in quasi-periodic and aperiodic plasmon lattices," Optica 3, 686 (2016).

66. F. Pratesi, M. Burresi, F. Riboli, K. Vynck, and D. S. Wiersma, "Disordered photonic structures for light harvesting in solar cells," Opt. Express 21, A460 (2013).

67. Y. J. Donie, M. Smeets, A. Egel, F. Lentz, J. B. Preinfalk, A. Mertens, V. Smirnov, U. Lemmer, K. Bittkau, and G. Gomard, "Light trapping in thin film silicon solar cells via phase separated disordered nanopillars," Nanoscale 10, 6651-6659 (2018).

68. Y. Zhang, Y. Xu, S. Chen, H. Lu, K. Chen, Y. Cao, A. E. Miroshnichenko, M. Gu, and X. Li, "Ultra-Broadband Directional Scattering by Colloidally Lithographed High-Index Mie Resonant Oligomers and Their EnergyHarvesting Applications," ACS Appl. Mater. \& Interfaces 10, 1677616782 (2018).

69. V. A. Markel and A. K. Sarychev, "Propagation of surface plasmons in ordered and disordered chains of metal nanospheres," Phys. Rev. B 75, 085426 (2007).

70. F. Rüting, "Plasmons in disordered nanoparticle chains: Localization and transport," Phys. Rev. B 83, 115447 (2011).

71. S. V. Karpov, I. L. Isaev, A. P. Gavrilyuk, V. S. Gerasimov, and A. S. Grachev, "Defects of colloidal crystals," Colloid J. 71, 329-339 (2009).

72. S. V. Karpov, I. L. Isaev, V. S. Gerasimov, and A. S. Grachev, "Effect of defects of plasmon resonance colloidal crystals on their extinction spectra," Opt. Spectrosc. 109, 372-378 (2010).

73. P. Moitra, B. A. Slovick, Z. Gang Yu, S. Krishnamurthy, and J. Valentine, "Experimental demonstration of a broadband all-dielectric metamaterial perfect reflector," Appl. Phys. Lett. 104, 171102 (2014).

74. T. J. Arruda, A. S. Martinez, and F. A. Pinheiro, "Controlling optical memory effects in disordered media with coated metamaterials," Phys. Rev. A 98, 043855 (2018).

75. M. Yannai, E. Maguid, A. Faerman, Q. Li, J.-H. Song, V. Kleiner, M. L. Brongersma, and E. Hasman, "Order and Disorder Embedded in a Spectrally Interleaved Metasurface," ACS Photonics p. acsphotonics.8b01138 (2018).

76. A. Rahimzadegan, D. Arslan, R. N. S. Suryadharma, S. Fasold, M. Falkner, T. Pertsch, I. Staude, and C. Rockstuhl, "Disorder-Induced Phase Transitions in the Transmission of Dielectric Metasurfaces," Phys. Rev. Lett. 122, 015702 (2019).

77. T. Huang, B. Wang, and C. Zhao, "Negative refraction in metamaterials based on dielectric spherical particles," J. Quant. Spectrosc. Radiat. Transf. 214, 82-93 (2018)

78. A. E. Ershov, I. L. Isaev, P. N. Semina, V. A. Markel, and S. V. Karpov, "Effects of size polydispersity on the extinction spectra of colloidal nanoparticle aggregates," Phys. Rev. B - Condens. Matter Mater. Phys. 
85, 1-10 (2012).

79. G. W. Mulholland, C. F. Bohren, and K. A. Fuller, "Light Scattering by Agglomerates: Coupled Electric and Magnetic Dipole Method," Langmuir. 10, 2533-2546 (1994).

80. O. Merchiers, F. Moreno, F. González, and J. M. Saiz, "Light scattering by an ensemble of interacting dipolar particles with both electric and magnetic polarizabilities," Phys. Rev. A - At. Mol. Opt. Phys. 76, 1-12 (2007).

81. C. F. Bohren and D. R. Huffman, Absorption and Scattering of Light by Small Particles (Wiley-VCH Verlag GmbH, Weinheim, Germany, 1998).

82. V. E. Babicheva and A. B. Evlyukhin, "Resonant Lattice Kerker Effect in Metasurfaces With Electric and Magnetic Optical Responses," Laser \& Photonics Rev. 1700132, 1700132 (2017).

83. A. García-Etxarri, R. Gómez-Medina, L. S. Froufe-Pérez, C. López, L. Chantada, F. Scheffold, J. Aizpurua, M. Nieto-Vesperinas, and J. J. Sáenz, "Strong magnetic response of submicron Silicon particles in the infrared," Opt. Express 19, 4815 (2011).

84. D. Smirnova, A. I. Smirnov, and Y. S. Kivshar, "Multipolar secondharmonic generation by Mie-resonant dielectric nanoparticles," Phys. Rev. A 97, 013807 (2018).

85. C. Zhang, Y. Xu, J. Liu, J. Li, J. Xiang, H. Li, J. Li, Q. Dai, S. Lan, and A. E. Miroshnichenko, "Lighting up silicon nanoparticles with Mie resonances," Nat. Commun. 9, 2964 (2018).

86. P. D. Terekhov, K. V. Baryshnikova, Y. A. Artemyev, A. Karabchevsky, A. S. Shalin, and A. B. Evlyukhin, "Multipolar response of nonspherical silicon nanoparticles in the visible and near-infrared spectral ranges," Phys. Rev. B 96, 035443 (2017).

87. E. D. Palik, Handbook of optical constants of solids /I (Academic Press, New York, 1998).

88. J. R. Allardice and E. C. Le Ru, "Convergence of Mie theory series: criteria for far-field and near-field properties," Appl. Opt. 53, 7224 (2014).

89. S. Rodriguez, M. Schaafsma, A. Berrier, and J. Gómez Rivas, "Collective resonances in plasmonic crystals: Size matters," Phys. B: Condens. Matter 407, 4081-4085 (2012).

90. L. Zundel and A. Manjavacas, "Finite-size effects on periodic arrays of nanostructures," J. Physics: Photonics 1, 015004 (2019).

91. D. G. Baranov, D. A. Zuev, S. I. Lepeshov, O. V. Kotov, A. E. Krasnok, A. B. Evlyukhin, and B. N. Chichkov, "All-dielectric nanophotonics: the quest for better materials and fabrication techniques," Optica 4, 814 (2017). 